\title{
ESTUDO FITOQUÍMICO E ANTIMICROBIANO DAS FOLHAS DE EUGENIA ASTRINGENS CAMBESS. (MYRTACEAE)
}

\author{
Bruna Carminate ${ }^{1}$ \\ Caroline Carminate ${ }^{2}$ \\ Andriely Armini Fontana ${ }^{3}$ \\ Maria Heloísa Almeida Farias ${ }^{4}$ \\ Marcelo Barreto da Silva ${ }^{5}$
}

Resumo: O conhecimento sobre plantas medicinais simboliza muitas vezes o único recurso terapêutico de muitas comunidades e grupos étnicos. A tradição popular é a origem de valiosos conhecimentos acerca das plantas, muitas das observações populares sobre a utilização de plantas medicinais contribuem, de forma relevante, para a divulgação das virtudes terapêuticas vegetais. $A$ E. astringens é uma espécie que apresenta casca e folhas aromáticas, adstringentes, anti-reumáticas e diuréticas. Apesar do uso medicinal já relatado, não existem muitos estudos quanto a atividade antimicrobiana. O objetivo do presente trabalho foi a realização da triagem fitoquímica e a avaliação da atividade antimicrobiana do extrato hidroalcoólico das folhas de Eugenia astringens Cambess. Foram encontrados taninos, fenóis, catequinas e ácidos orgânicos e o extrato apresentou atividade antibacteriana para todas as 8 cepas testadas.

Palavras-chave: Eugenia astringens; Myrtaceae; Antibióticos; Bactérias.

\footnotetext{
${ }^{1}$ Agronomia/Universidade Federal do Espírito Santo, Centro Universitário Norte do Espirito Santo, Brasil. E-mail: brunabcarminate@hotmail.com.

${ }^{2}$ Odontologia/Faculdade Espirito Santense - FAESA, Brasil. E-mail: carolinebcarminate@gmail.com.

${ }^{3}$ Farmácia/Universidade Federal do Espírito Santo, Centro Universitário Norte do Espirito Santo, Brasil. E-mail: andrielyfontana@hotmail.com.

${ }^{4}$ Farmácia/Universidade Federal do Espírito Santo, Centro Universitário Norte do Espirito Santo, Brasil. E-mail: mariaheloisamh@hotmail.com.

${ }^{5}$ Professor Titular/Universidade Federal do Espírito Santo, Centro Universitário Norte do Espirito Santo, Brasil. E-mail: marcelobarretodasilva@gmail.com.
} 